\title{
Transdermal delivery of tolterodine tartrate for overactive bladder treatment: In vitro and in vivo evaluation
}

\author{
RAJAN RAJABALAYA ${ }^{1,2}$ \\ CHUNG YEE MUN ${ }^{2}$ \\ JESTIN CHELLIAN ${ }^{2}$ \\ SRIKUMAR CHAKRAVARTHI ${ }^{2,3}$ \\ SHEBA R. DAVID ${ }^{* 1,2}$ \\ ${ }^{1}$ PAPRSB Institute of Health Sciences \\ Universiti Brunei Darussalam \\ Bandar Seri Begawan BE1410 \\ Brunei Darussalam \\ ${ }^{2}$ School of Pharmacy, International \\ Medical University, Bukit Jalil 57000 \\ Kuala Lumpur, Malaysia \\ ${ }^{3}$ School of Medicine, Perdana \\ University, Jalan MAEPS Perdana \\ 43400 Serdang, Selangor, Malaysia
}

Accepted May 11, 2017

Published online June 1, 2017

\begin{abstract}
The purpose of the study was to develop a transdermal tolterodine tartrate (TT) patch and to analyse its efficacy for overactive bladder (OAB) treatment. Patches were prepared using various polymers and plasticizers via the solvent casting method. The patches were characterized for tensile strength, thickness, moisture content, modulus of elasticity and water absorption capacity. Differential scanning calorimetry and Fourier transform infrared analyses were also performed. To determine patch effectiveness, in vitro release, permeation and animal studies were performed. The patches showed satisfactory percentage of release, up to $89.9 \%$, and their mechanical properties included thickness (0.10-0.15 mm), tensile strength (4.62-9.98 MPa) and modulus of elasticity (20-29 MPa). There were no significant interactions between TT and other excipients. Animal studies indicated that the TT patch reduced the incidence of side effects; however, studies of longer duration are required to determine the effectiveness in treating OAB.
\end{abstract}

Keywords: tolterodine tartrate, transdermal, overactive bladder, matrix patches, permeation

The International Continence Society (ICS) defined overactive bladder (OAB) as ,a collection of symptoms suggestive of lower urinary tract dysfunction such as urgency, with or without urge incontinence, normally accompanied with frequency and nocturia" (1). The National Overactive Bladder Evaluation (NOBLE) study reported that OAB affects approximately $16 \%$ of the population (2) and affects the quality of life of patients. Pharmacological anti-muscarinic molecules like trospium, oxybutynin and tolterodine tartrate (TT) are used in the treatment of this chronic condition (3). However, oral administration exposes patients to side effects such as headache, constipation and dry mouth (4). To minimize these side effects and to improve compliance, transdermal delivery is a promising alternate route (5). This non-selective, competitive anti-muscarinic agent, TT, is recommended as a first-line pharmacological treatment for OAB (6). Upon administration, TT is metabolized by the liver (Cytochrome P450 2D6) to pharmacologically active 5-hydroxy-

\footnotetext{
* Correspondence; e-mail: sdsheba@gmail.com
} 
methyl derivative $(7,8)$. The drug has in vivo functional selectivity for muscarinic receptors in the bladder over the salivary glands and hence it is suitable for the treatment of OAB $(9$, 10). Transdermal delivery allows superior control of systemic drug concentration, by-passes first pass metabolism, minimizes gastrointestinal side effects, allows immediate termination of administration in case of toxicity and greatly improves patient compliance (1113). Zhao reported that the flux of TT solution was not affected by addition of $O$-acylmenthol derived chemical enhancers but skin reservoir effects of enhancer-containing groups showed promising results compared to the control group (14). Choi reported that free base TT penetrated hairless mouse skin about 10 times more effectively compared to other lipophilic salt forms (15). Elshafeey et al. developed controlled release of the drug into the systemic circulation with the use of TT microemulsion, which was advantageous in prevention of nocturnal enuresis with improvement in patient compliance (16). Similar studies of both oxybutynin and TT proniosomal gel were reported by our group to be suitable for transdermal route, which reduced the dry mouth effect compared to the oral route in our earlier studies $(17,18)$. The drug had not been explored for transdermal delivery as a patch and this is the first study that analyses the efficacy of this formulation. The main aim of this research is to formulate a TT transdermal matrix patch possessing desirable physicochemical, release and permeation characteristics with reduced side effects as well as increased efficacy for OAB.

\section{EXPERIMENTAL}

\section{Materials}

TT was a gift from Aurobindo Pharmaceuticals (India). Eudragit E 100, RS100, RL100, RSPO and RLPO were gifts from Evonik Röhm GmbH, Germany. EUDRAGIT ${ }^{\circledR}$ polymers are copolymers derived from esters of acrylic and methacrylic acid whose physicochemical properties are determined by functional groups. In these studies, Eudragit E 100 is a cationic polymer with dimethylaminoethyl methacrylate as a functional group. Other Eudragit grades such as RS100, RL100, RSPO and RLPO are ethyl acrylate, methyl methacrylate copolymers with trimethyl-ammonioethylmethacrylate as different ratios of the functional group. Polyvinyl pyrrolidone (Kollidon ${ }^{\circledR} 30$ ) was procured from the BASF Chemical Company, Germany. Dibutyl phthalate (DBP) was purchased from Qualigens Fine Chemicals, India. Triethyl citrate (TEC) was purchased from Merck Chemicals, Germany. Dibutyl Sebacate (DBS) was purchased from Sigma-Aldrich Chemie GmbH, Germany. All other chemicals used in the study were of analytical grade and were used as received.

\section{Preparation of patches}

The polymeric matrix formulation was prepared by dissolving different grades ( $\mathrm{E}$ 100, RSPO and RLPO) of Eudragit polymers (200-400-600 mg) in chloroform, then $100 \mathrm{mg}$ PVP was added under uniform stirring for 15-20 min. The patches were formed using the solvent casting method. To the above solution, one of the plasticizers (DBS/DBP/TEC) at either 10 or $15 \%$ of total polymer mass and $8 \mathrm{mg}$ of TT were added at room temperature under uniform slow stirring. The resulting solution was poured into casts that had an aluminium foil backing layer. It was dried for $24-48 \mathrm{~h}$ at room temperature (19). Table I con- 
tains details of the various formulation combinations. All formulations with the codes starting with A (A1, A2, A3) were designated as series A. All other series were similarly categorised into series $\mathrm{B}$ to $\mathrm{F}$.

\section{CHARACTERISATION OF TRANSDERMAL PATCHES}

\section{Patch thickness and tensile strength}

A digital micrometer (Mitutoyo, Japan) was used for determining patch thickness (Table I). Tensile strength was measured with a load of $50 \mathrm{kN}$ at $5 \mathrm{~mm} / \mathrm{min}$ extension speed using an Instron 4204, UK tensilometer. The test was performed according to the D $882-$ $75 \mathrm{D}$ method of the American Society for Testing Materials (ASTM) for 6 samples of each formulation patch. Temperature of $25 \pm 2{ }^{\circ} \mathrm{C}$ and humidity of $56 \pm 2 \%$ were maintained throughout the test (19).

Equation (1) was used for tensile strength calculation.

$$
\tau=\frac{L_{\max }}{A_{\mathrm{i}}}
$$

where $\tau$ is tensile strength; $L_{\max }$ is maximum load and $A_{\mathrm{i}}$ is the initial cross-sectional area of the sample.

\section{Modulus of elasticity determination}

The modulus of elasticity reflects the stiffness or elasticity of transdermal patches. This indicates the resistance to distortion of the films and was calculated by plotting the stress strain curve using an Instron 4204, UK tensilometer. The modulus of elasticity is represented as the ratio of applied stress over strain in the region of elastic deformation and was determined using the following formula (2):

$$
E=\text { stress/strain }
$$

\section{Moisture content}

Matrix patches were independently weighed and preserved in a desiccator for a day at $40{ }^{\circ} \mathrm{C}$. They were consecutively reweighed until a constant mass was established. The initial and constant final patch mass difference was noted to calculate the moisture content percentage. Six readings were recorded and the average was calculated as described by Rajabalaya et al. (19).

\section{Water absorption}

Water absorption capacity was determined by weighing the dry patches initially. They were then kept at two different relative humidities for 24 hours at room temperature. Saturated sodium chloride solution and saturated ammonium hydrogen phosphate solution had yields of 75 and $93 \%$ humidity, respectively. Patch mass was recorded periodically until it was constant. Average of six readings was recorded (Table I) (19). 


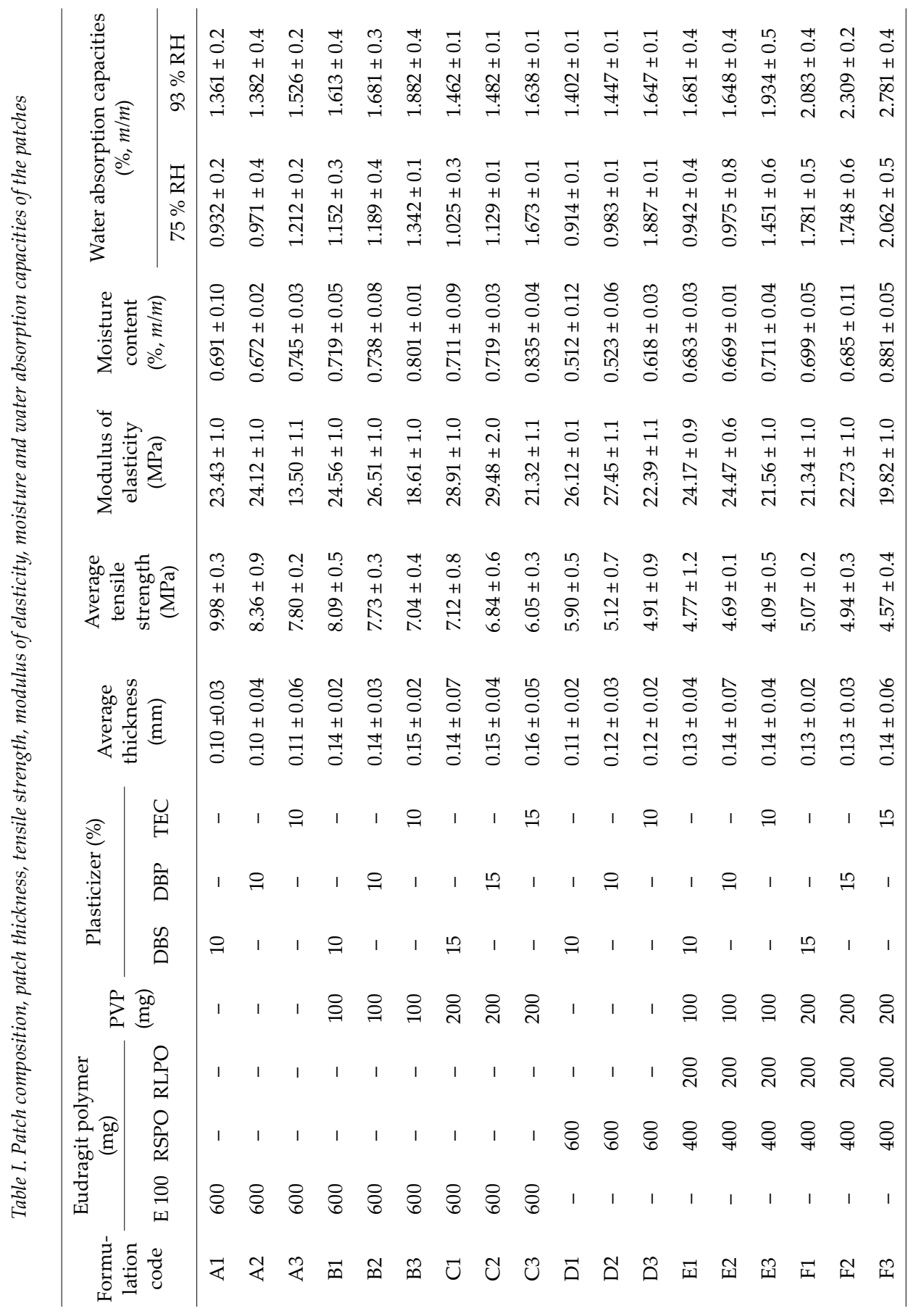




\section{Fourier Transform Infrared (FTIR) studies}

Tolterodine tartrate, PVP, E 100 and their combination are depicted in Fig. 1a, while TT, RLPO, RSPO, PVP, TT and their combination are represented in Fig. 1b. Each component was mixed individually with $\mathrm{KBr}$ (IR grade) in the 100:1 ratio and pressurized using a hydraulic press to obtain pellets. These were scanned using an FTIR spectrophotometer in the range of $4000-400 \mathrm{~cm}^{-1}$ (FT-IR 8400S, Shimadzu, Japan).

\section{In vitro release studies}

Franz (vertical) Diffusion Cells (PermeGear, USA) were used for in vitro studies. A 1.7 $\mathrm{cm}^{2}$ diameter matrix patch was placed carefully, with the backing membrane facing the atmosphere, on the receptor compartment that contained phosphate buffered saline (PBS) at $\mathrm{pH}$ 7.4. The assembly was maintained at a uniform temperature of $32 \pm 0.5^{\circ} \mathrm{C}$ with uniform stirring speed of $50 \mathrm{rpm}$. Diffusion of the drug from the matrix patch was ensured by placing the drug side in contact with PBS in the receptor compartment. Every hour, 1 $\mathrm{mL}$ of the receptor sample was withdrawn and the same volume of buffer solution was replaced. The sample was measured using reverse-phase HPLC (1200 HPLC series; Agilent Technologies, Santa Clara, CA, USA) with a C-18 analytical column $(5 \mu \mathrm{m}, 4.6 \times 150 \mathrm{~mm}$; Phenomenex, Torrance, CA, USA). The sample was measured in UV at a $281 \mathrm{~nm}$ wavelength with $35: 65, V / V$ of mobile phase $(20 \mathrm{mM}$ pH 3.0 acetate buffer/methanol) at an elu-

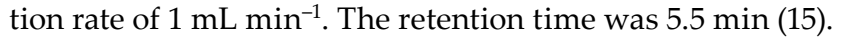

\section{Release kinetics data treatment}

Various kinetic models, zero order, first order Higuchi and Korsmeyer-Peppas were applied to the in vitro release data. These models serve to predict the release mechanism and kinetics of the drug from the transdermal matrix patch (Table II).

\section{In vitro permeation studies}

Sprague Dawley rats $(n=6)$ were used in the study. Each rat was sacrificed as per the cervical dislocation method protocol. A section of the freshly excised abdominal rat skin treated in isotonic solution was bound intimately with a transdermal patch to the donor compartment without any air gap between the drug side of the patch and the stratum corneum side of the skin. The dermal side of the skin just touched the receptor liquid surface horizontally to allow drug permeation. The receptor compartment temperature, volume of sample collection and method of analysis were similar to the in vitro release study $(17,20)$. The International Medical University (IMU) Research Ethics Committee (B0109_ Res182012) approved the procedures and animal care for the experiments undertaken in this project including pharmacological experiments. Percentage of permeation and permeation rate were calculated and are given in Table III.

\section{In vivo animal studies}

\section{Acetic acid (ACA)-induced bladder hyperactivity}

Male Sprague Dawley (SD) rats, initial mass 350-450 g, were used in the experiment. Rats were housed in the Animal Holding Facility (AHF) of the International Medical Uni- 
versity (IMU). They were housed in plastic cages, 3 rats per cage. The environment was maintained at ambient temperature with a $12 \mathrm{~h}$ light/12 h dark cycle. All rats were allowed free access to normal commercial rat feed pellets (Gold Coin Feedmills, Malaysia Sdn. Bhd) and reverse osmosis $(\mathrm{RO})$ water. Rats were acclimatized for one week prior to experimental protocols. All the experimental procedures were performed in accordance with the guidelines issued by the International Medical University Joint Committee (IMU-JC) for Ethics.

Bladder inflammation caused by the administered ACA solution $(0.75 \%)$ was evaluated histopathologically. A urinary catheter was used to administer the solution to unconscious rats. This model produces micturition due to the acute inflammation caused in the urinary bladder by ACA (21).

\section{Measurement of salivary secretion}

The experiment was conducted on diethyl ether anesthetized rats (21). Cotton balls (3-5) were kept for 10 mins for saliva absorption; they were immediately weighed to avoid moisture loss. Both oral and patch treatments were given at 0 hour along with the pilocarpine injection. The amount of saliva collected in the cotton balls was measured at 2, 4, 12 and $24 \mathrm{~h}(17,21)$.

\section{Histological studies}

After the treatment period, each rat was anaesthetized and sacrificed as per protocol. The bladder was reached after dissecting the abdomen wall and exposing the peritoneum space. The bladder dome was dissected down to the level of ureteral insertion. The excision was done as a total cystectomy procedure involving ligating at the level of pelviureteric junction above and urethra below. This ensured that all 3 layers, urothelium, lamina propria and muscle layers were intact for the study. Bladders were fixed in $10 \%$ buffered formalin overnight, processed, embedded in paraffin, and cut into $5-\mu \mathrm{m}$ slices. All staining was done on at least two different areas of the bladder. These were then observed under a light microscope after hematoxylin and eosin staining (17).

\section{Statistical analyses}

SPSS v18.0 (IBM Corp., USA) statistical software was used for data analysis. Paired $t$ test and analysis of variance were both performed. $p<0.05$ was considered significant. Values are expressed as mean \pm standard deviation.

\section{RESULTS AND DISCUSSION}

Different grades of Eudragit polymers, PVP, different categories of plasticizers, such as dibutyl sebacate (DBS), dibutyl phthalate (DBP) and triethyl citrate (TEC), used for the preparation of patches are presented in Table I. Mechanical properties of the matrix patch such as modulus of elasticity, tensile strength and thickness are also given in Table I.

\section{Thickness and tensile strength}

TEC is the most hygroscopic plasticizer and is followed by DBP and DBS; therefore, patches containing TEC (A3) had the highest thickness amongst series A, with E 100 fol- 
lowed by DBP and DBS containing patches (22). Correspondingly, B3 and C3 had the highest thickness in their respective series. Likewise, similar observations were made with other Eudragit polymer grades (RSPO/RLPO) of D and E series patches. A tensile strength of 4 $\mathrm{MPa}$ and above is desirable for transdermal patches according to the American Society for Testing and Materials (ASTM) guidelines (23). The data revealed that the average tensile strength of all the patches was in the range of 4.09 to $9.98 \mathrm{MPa}$, indicating good tensile strength. This can be illustrated with E3 having 4.09 MPa, which is the least in series E (22). DBS patches (Ex: A1 - 9.98 MPa) always maintained the highest tensile strength among their respective series compared to those of the other two plasticizers, while patches with DBP exhibited a higher modulus of elasticity (22.73 to $29.48 \mathrm{MPa}$ ) than the other two plasticizers.

Patch thickness increased in the formulations containing hydrophilic polymers such as PVP, RLPO and Eudragit RL100, as evidenced in Table I. Patch thickness in Eudragit E 100 series is the lowest compared to other series patches containing an additional PVP (B and C series). In contrast, the results of tensile strength of matrix patches decreased with an increase in the amount of PVP in the patches. Increase in the percentage of plasticizer from 10 to $15 \%$ reduced the tensile strength of patches, as observed in the $\mathrm{C}$ and $\mathrm{F}$ series. The hygroscopic nature of PVP may have increased the matrix patch bulkiness in the B and $C$ series, as witnessed by lesser patch thickness with $100 \mathrm{mg}$ PVP compared to patches with $200 \mathrm{mg}$ (24). Similarly, the hygroscopic nature of the plasticizer was also responsible for higher thickness of the patches.

The tensile strength data supports the possibility of polymer-polymer bond strength decrease due to the disruption in continuity of polymer chain molecules (19). The molecular weight of the plasticizer also affects the tensile strength of matrix patches (25). The descending order of plasticizer molecular weight used in this study is DBS $>$ DBP $>$ TEC. Low molecular weight plasticizers increase the plasticizing effect by increasing the space between the polymeric chains, thus reducing the tensile strength. Optimal tensile strength for the patch is necessary to keep the patch on the skin and withstand the movements of daily life. The prepared patches had the necessary tensile strength for everyday wear.

\section{Moisture content and water absorption studies}

Moisture content of patches with hydrophilic excipients (PVP and RLPO) was higher compared to that of hydrophobic nature polymeric patches ( E 100 and RSPO). Matrix patches with TEC (A3, B3, C3, D3, E3 and F3) exhibited increased water absorption capacity in both 75 and $93 \%$ RH due to the hygroscopic nature of TEC. Higher amount of PVP (200 $\mathrm{mg}$ ) accounts for the higher moisture content of the PVP containing patches, namely, F3 with $0.881 \%(\mathrm{~m} / \mathrm{m})$. Both B and C series had lower and higher PVP content, respectively, and exhibited proportionate water absorption capacities with B having lower capacity. A similar pattern was displayed by the E and F series (22). Hydrophilic plasticizer TEC was also responsible for higher moisture uptake and water absorption capacity. Moisture content and water absorption capacities of the E 100 patches were dependent on the concentration of plasticizer and PVP used in the study (26). Water absorption under both of the relative humidity conditions was higher with higher PVP contents, because it allowed water molecules to easily migrate into the matrix system, which led to increased moisture uptake as well as absorption of water (25). Hydrophobic DBS containing patches are tough to hydrate, especially at $15 \%$. It is clear from Table I that the patches remained intact and stable during and after water uptake capacity studies. 


\section{Fourier Transform Infrared (FTIR) studies}

FTIR spectra (Fig. 1a,b) of individual polymers (Eudragit E 100, RSPO and RLPO) and PVP and TT including a combined mixture of E 100/PVP/TT; RSPO/RLPO/TT were compared for changes in spectral shifts in the mixture. FTIR spectra of the E 100/PVP, RSPO/ RLPO and the drug TT combined mixture are shown in Fig. 1a,b. All peaks of the polymers used in the mixture were observed in the FTIR spectra. FTIR spectra of the drug and combined mixtures revealed the following bands:-OH stretching at $3437.50 \mathrm{~cm}^{-1},-\mathrm{C}-\mathrm{H}$ stretching (aromatic) at 3050.83 and $3027.33 \mathrm{~cm}^{-1},-\mathrm{CH}_{3}$ stretching (aliphatic) resulting in 2695.11 and $2924.92 \mathrm{~cm}^{-1}, \mathrm{C}=\mathrm{C}$ stretching (aromatic) resulting in 1600.34 and $1452.95 \mathrm{~cm}^{-1}$. Polymer and drug compatibility is of the paramount importance. The FTIR study clearly showed that there were no significant spectral shifts in peaks corresponding to different Eudragit polymers or the drug (19). There were, however, a few overlaps in characteristic peaks of the drug and the polymer. This confirms that there were no interactions and indicates the compatibility of the drug with other components.

In vitro release studies

The 8-h cumulative release (\%) of the matrix patches is shown in Fig. 2. The figure depicts an increase in the release percentage with an increase in matrix patch plasticiser percentage for the DBS containing patches. However, in the case of E 100 based matrix

a)

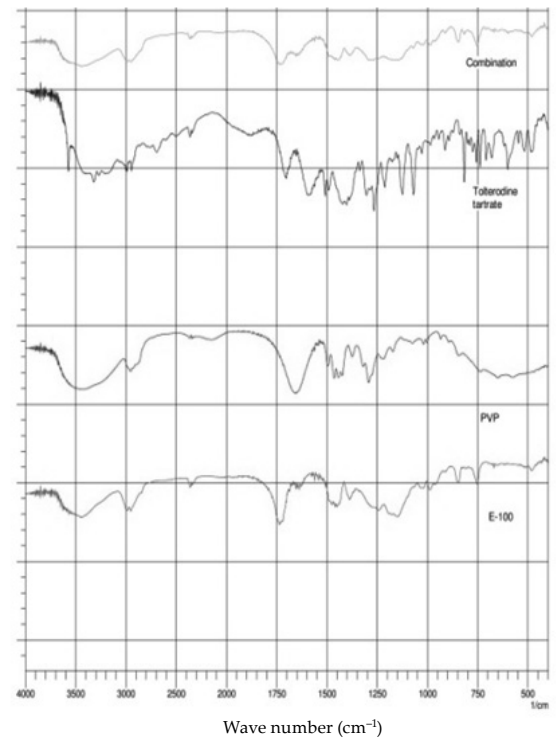

b)

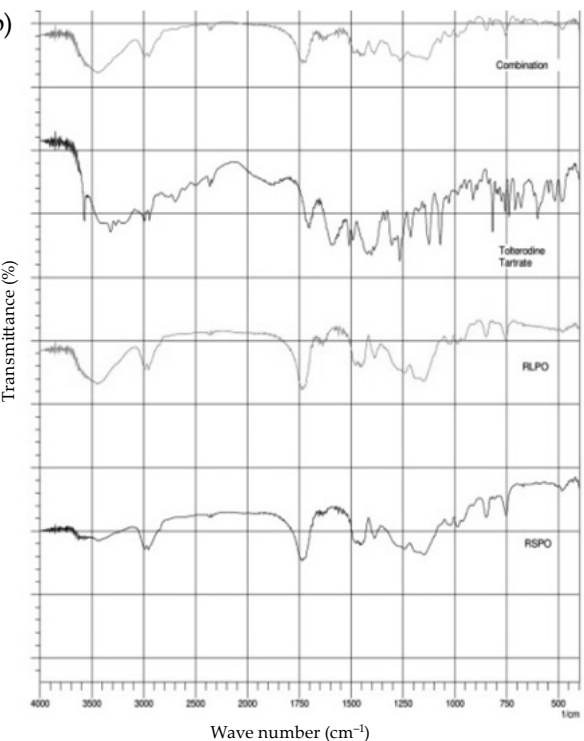

Fig. 1. FTIR spectra for: a) E 100, PVP, tolterodine tartrate and combination, b) RLPO, RSPO, tolterodine tartrate and combination. 
patches containing DBS or DBP as plasticizer, the release was significantly lower without PVP (A1, 22.82 \%) compared to that of the PVP comprising patch (B1, 33.65 \% and C1, 89.90 $\%$ ). Series D, E and F (D1, E1 and F1) containing RSPO and RLPO polymers with DBS as plasticizer (D1, $48 \%$, E1, $79.7 \%$ and F1, 74.6 \%) showed a higher percentage of drug release compared to the DBP patches (D2, $34.3 \%$, E2, $40.5 \%$ and F2, $53 \%$ ). The TEC containing patches (D3, $44.64 \%$, E3, $41.09 \%$ and F3, $49.03 \%$ ) had higher drug release compared to (D2, E2 and F2) DBP containing patches. In vitro release studies were conducted to ascertain the drug diffusion pattern from the matrix patches. The following interesting discussion could be drawn from the observed results. The percentage of release of TT was higher with PVP containing patches, this may be due to the dissolution of hydrophilic PVP in the buffer medium, forming pores on the matrix patch and leaching the drug from the formulation into the solution, thus increasing the rate of diffusion into the patch (19).

It is not only the PVP but also the plasticizer that influences the drug release behaviour in the transdermal delivery system from matrix patches. An increase in the number of plasticizer molecules between polymer chains reduces the inter-molecular attractive forces between the polymer chains, thus leading to easy diffusion out of the matrix patch. According to the report by Siepmann et al. (27), while DBS is the right plasticizer for fast release, DBP should be selected for more prolonged drug release. The hydrophilic TEC plasticizer tends to absorb moisture and leach it out from the matrix patch, leading to disruption of polymeric chain integrity resulting in pores and reduction of plasticizer content, which increases drug diffusion out of the matrix patch (22); in contrast, DBP is hydrophobic and yields the opposite effect.

\section{Release kinetics data treatment}

As regards release kinetics, the best-fit was found with Higuchi's model, which calculates mass transport processes using the first-order release kinetics equation. All the nvalues for formulations containing plasticizer TEC exceeded 0.89 , which suggests either

a)

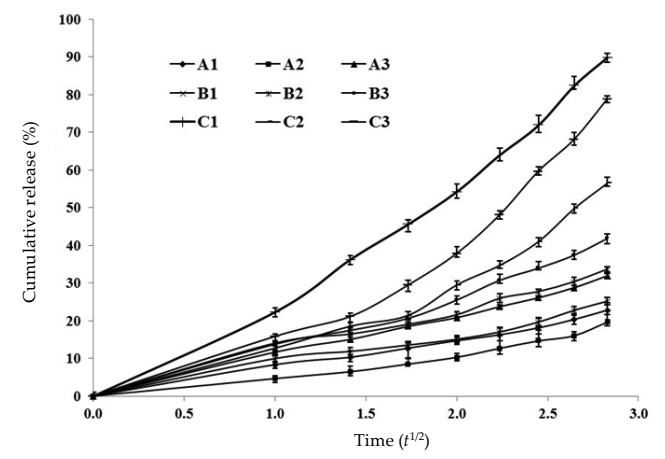

b)

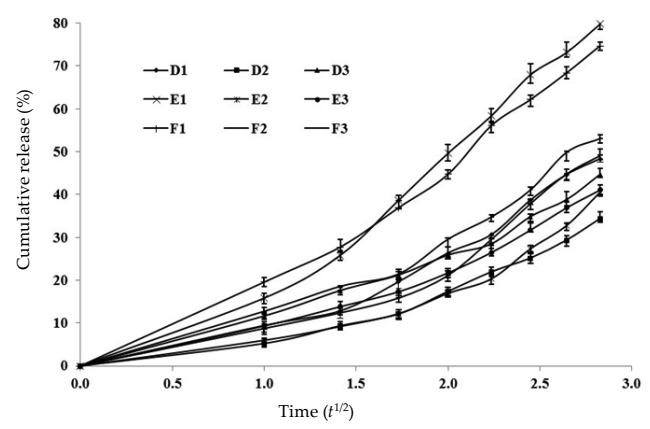

Fig. 2. a) Release profiles of series A, B and C, b) release profiles of series D, E and F. 
R. Rajabalaya et al.: Transdermal delivery of tolterodine tartrate for overactive bladder treatment: In vitro and in vivo evaluation, Acta Pharm. 67 (2017) 325-339.

Table II. Release kinetics data treatment

\begin{tabular}{lccccccccc}
\hline Model & A1 & A2 & A3 & B1 & B2 & B3 & C1 & C2 & C3 \\
\hline Zero order & 0.9524 & 0.9679 & 0.9901 & 0.9649 & 0.9402 & 0.9901 & 0.9239 & 0.9919 & 0.9922 \\
First order & 0.9662 & 0.9775 & 0.9925 & 0.9824 & 0.9558 & 0.9727 & 0.9720 & 0.9790 & 0.9125 \\
Higuchi & 0.9856 & 0.9818 & 0.9231 & 0.9810 & 0.9817 & 0.8653 & 0.9971 & 0.8979 & 0.9062 \\
Korsmeyer Peppas & 0.9900 & 0.9926 & 0.9913 & 0.9847 & 0.9727 & 0.9865 & 0.9954 & 0.9942 & 0.9949 \\
$n$ value & 0.6600 & 0.6110 & 0.9650 & 0.6426 & 0.5676 & 1.0710 & 0.4998 & 0.1052 & 0.9431 \\
Model & $\mathrm{D} 1$ & $\mathrm{D} 2$ & $\mathrm{D} 3$ & $\mathrm{E} 1$ & $\mathrm{E} 2$ & $\mathrm{E} 3$ & $\mathrm{~F} 1$ & $\mathrm{~F} 2$ & F3 \\
Zero order & 0.8313 & 0.7751 & 0.8978 & 0.9094 & 0.9186 & 0.8956 & 0.9695 & 0.9764 & 0.9332 \\
First order & 0.8564 & 0.8156 & 0.9128 & 0.9330 & 0.9171 & 0.9329 & 0.9116 & 0.9680 & 0.9672 \\
Higuchi & 0.9092 & 0.9510 & 0.9341 & 0.9910 & 0.9276 & 0.9661 & 0.9363 & 0.9667 & 0.9800 \\
Korsmeyer Peppas & 0.8818 & 0.9430 & 0.8956 & 0.8460 & 0.9820 & 0.8679 & 0.9169 & 0.9814 & 0.9455 \\
$n$ value & 0.6678 & 0.3736 & 0.4857 & 0.9857 & 0.4800 & 0.4359 & 0.9951 & 0.6807 & 0.4774 \\
\hline
\end{tabular}

Case-2 relaxation or super Case-2 transport of polymeric chain erosion (Table II). The Higuchi model is established on the hypotheses that the initial drug concentration in patches is higher than its solubility in the receptor and that the swelling of matrix patches and dissolution rates are negligible (28). Results showed that formulations containing plasticizer DBS followed the Higuchi release model. According to the Higuchi model, the DBS formulations followed a diffusion controlled drug release mechanism.

\section{In vitro permeation studies}

Series $C$ and $E$ were selected for in vitro permeation studies based on the above excellent physicochemical, mechanical and in vitro release characteristics of transdermal patches. As shown in Fig. 3, formulations $\mathrm{C} 1$ and E1 showed the highest cumulative percentage of drug permeation, 63.07 and $63.25 \%$, respectively. The initial rapid release (burst effect) was due to rapid movement of the drug from the surface of the matrix. After the burst effect, the drug was released gradually at a regular pace, achieving a controlled release state. In vitro permeation study exhibited concentration dependent kinetics. Amongst all formulations, the two formulations tested for salivary secretion, the first with E 100 and higher PVP content and the second with the RSPO-RLPO combination with PVP (C1 and E1) showed rapid initial permeation (Fig. 3), which is desirable for transdermal delivery. Both parameters, the gradient of drug concentration as well as the vehicle-membrane partition coefficient were influenced by the extent of drug solubility in the vehicle $(16,19)$. The permeation percentage and permeation rate for the selected formulations ( $\mathrm{C} 1$ and $\mathrm{E} 1)$ showed higher rapid initial permeation and permeation rate (Table III: 4.23 and $3.47 \mu \mathrm{g} \mathrm{cm}^{-2} \mathrm{~h}^{-1}$, respectively) compared to other formulations. Hence, these two formulations were chosen for further in vivo animal studies. 


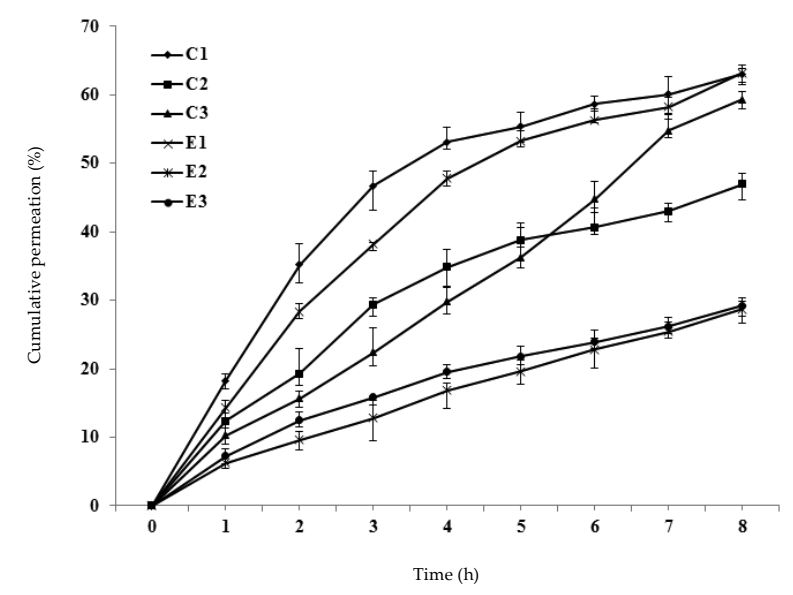

Fig. 3. Permeation profiles of series $\mathrm{C}$ and E.

\section{In vivo animal studies}

\section{Salivary secretion}

Based on the results of mechanical, physicochemical and in vitro release/permeation study, patches $\mathrm{C} 1$ and $\mathrm{E} 1$ were selected for the in vivo animal study. Whole saliva secreted in the oral cavity in 10 minutes was collected after 15 minutes of pilocarpine stimulation. After 1 and 12-hour administration of oral TT, there was a 1.68 and 2.39 fold decrease in whole saliva secretion, respectively, compared to control rats (Fig. 4a,b). This shows that oral TT markedly suppresses pilocarpine-stimulated salivation. Salivary secretion studies indicated

a)

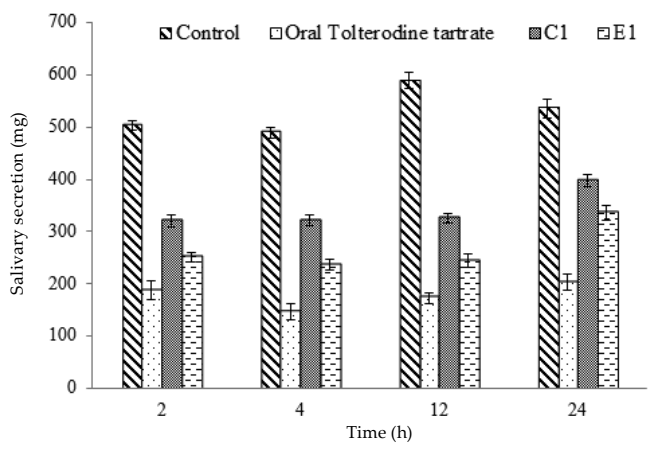

b)

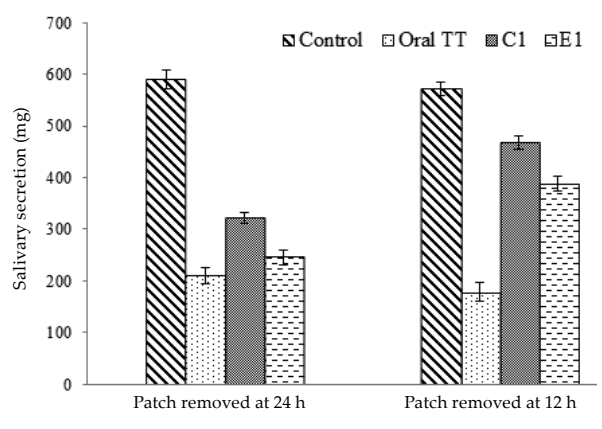

Fig. 4. Salivary secretion in rats with various formulations over 24 hours: a) control (no TT treatment) compared to oral, $\mathrm{C} 1$ and $\mathrm{E} 1$ formulations, b) recovery of pilocarpine-induced salivary secretion after gel formulation removal. 
Table III. In vitro permeation studies

\begin{tabular}{ccc}
\hline $\begin{array}{c}\text { Formulation } \\
\text { code }\end{array}$ & Permeation $(\%)$ & $\begin{array}{c}\text { Permeation rate } \\
\left(\mu \mathrm{g} \mathrm{cm}^{-2} \mathrm{~h}^{-1}\right)\end{array}$ \\
\hline C1 & 63.07 & 4.23 \\
C2 & 46.96 & 2.89 \\
C3 & 59.34 & 3.14 \\
E1 & 63.25 & 3.47 \\
E2 & 28.67 & 2.73 \\
E3 & 29.21 & 2.85 \\
\hline
\end{tabular}

higher activity of oral TT in the salivary gland than the transdermal matrix patch containing TT (10). A decrease was observed in the total amount of saliva collected in the second hour after patch administration, demonstrating that transdermal TT exerted a lesser effect on salivary glands than oral administration. The main salivary secretion of transdermal application produced lower suppression of pilocarpine-stimulated salivation. The results showed that the suppression of pilocarpine-stimulated salivation was greatly reduced after $12 \mathrm{~h}$ of removal of both of the matrix patch formulations. These observations demonstrate the advantages of the TT containing transdermal patch over oral formulation, leading to less dry mouth. In addition, controlled release from transdermal formulations for the muscarinic receptor resulted in longer duration of action, albeit with slower onset (29).

\section{Histopathology in the bladder inflammation model}

Fig. 5a shows the morphology of a healthy normal urinary bladder consisting of multilayers of transitional cell epithelium, sub-mucosa and muscle layers. Fig. $5 b$ and $5 c$ give the microscopic picture of the group treated with $0.75 \%$ acetic acid. Necrosis of the transitional cells and flat ulcer atrophy of the mucosa epithelium with the presence of neutrophils and lymphocytes suggest acute inflammation. This demonstrates that acetic acid has caused damage to the bladder. Fig. $5 \mathrm{~d}$ provides microscopic information for the group that was treated with acetic acid and received oral TT treatment. It shows near normal urinary bladder with no evidence of inflammatory cells, which confirms the positive healing effect of oral tolterodine. Fig. 5e and $5 \mathrm{f}$ give the microscope picture for groups treated with TT transdermal patches. Focal areas of necrosis and ulceration of the mucosa with mild to moderate inflammation in sub-mucosa indicates better healing process in $\mathrm{C} 1$ and slow healing in E1.

While the urinary bladder of the former was normal and intact, the latter displayed transitional cell necrosis, inflammatory infiltration and ulceration of mucosa, indicating acute cystitis. However, the oral TT treated animals had near normal structures in the bladder, indicating complete healing. Histopathology of the urinary bladder of the $\mathrm{C} 1$ treated group exhibited fast regeneration of the transitional epithelium while the E1 treated group with a similar bladder structure as C1 displayed mild ulceration in mucosa with scattered inflammation. However, slides from the transdermal matrix patch revealed inflammation in the bladder with mild to moderate inflammation in sub-mucosa indicating 
a)

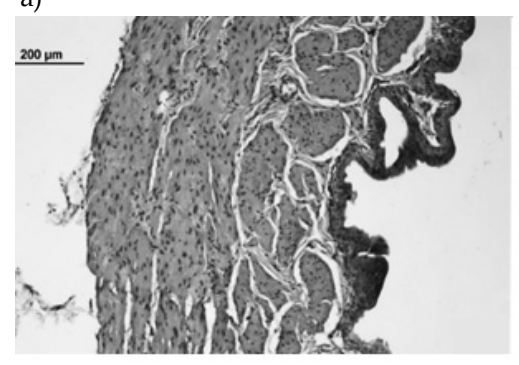

c)

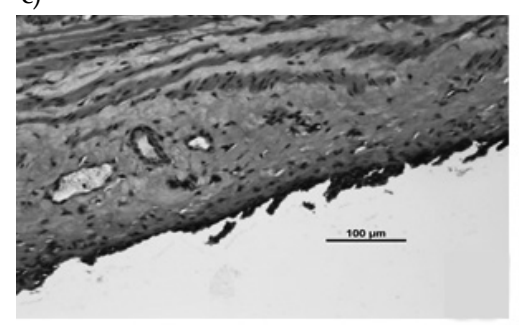

e)

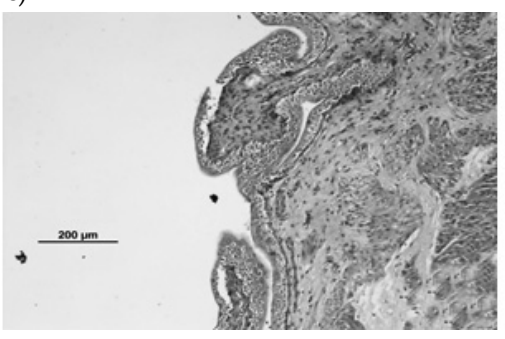

b)

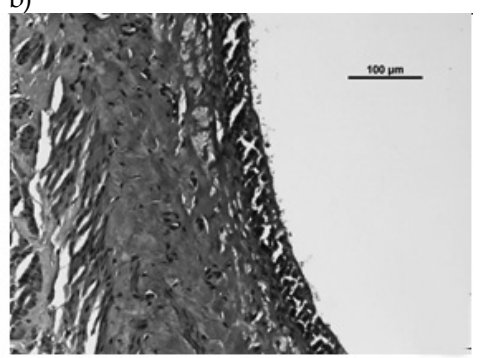

d)

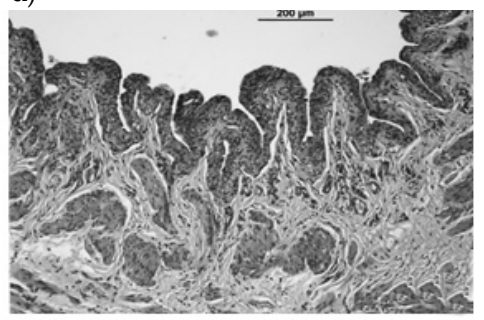

f)

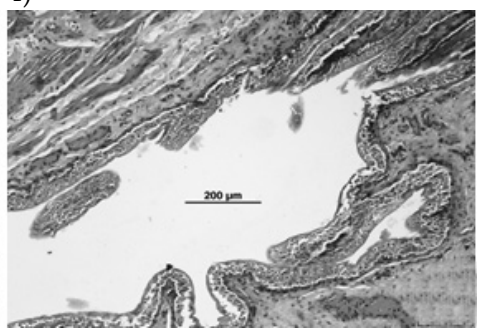

Fig. 5. Representative micrographs from rat bladders taken immediately after micturition studies: a) control (normal) group, b) acetic acid treated group showing edema, c) acetic acid treated group showing necrosis and ulceration, d) acetic acid treated group given TT orally, e) C1 patch, f) E1 patch formulations.

the beginning of the healing process. Nevertheless, it was slower than oral TT, which may be explained as follows: first, since transdermal patch formulation followed the controlledrelease pattern, it took time for the drug to reach the target site. Hence, a further 24-hour study is required to evaluate the complete healing effect. Second, the drug loading was insufficient to produce a significant therapeutic effect due to the smaller area available for patch application on rat skin compared to humans. Anyhow, drug loading can be augmented by increasing the drug concentration or patch application area.

\section{CONCLUSIONS}

Analysis of our experimental data findings suggest that, amongst the transdermal patches studied, the C1 patch (Eudragit E 100/PVP/DBP) had the most effective polymer 
and plasticizer combination. This polymeric matrix patch, which had the ideal physicochemical and mechanical and in vitro permeation profiles, could be applied directly to the skin for the OAB therapy. In vivo studies revealed that the $\mathrm{C} 1$ patch treated animals not only had higher salivary secretion but also achieved faster epithelial bladder regeneration compared to the E1 formulation. Thus, this study has established the effectiveness of transdermal TT matrix patch formulations in treating OAB in addition to reducing the dry mouth effects. Results also showed the feasibility of tolterodine tartrate transdermal delivery in OAB treatment. Further work on establishing the efficacy of therapeutic activity of the drug is to be carried out via pharmacokinetic and pharmacodynamic studies.

Acknowledgements. - The authors express their sincere gratitude to the Research Laboratory of the International Medical University, Kuala Lumpur, Malaysia, for providing financial support (Research grant No. B0109_Res182012) and laboratory facilities for the study.

\section{REFERENCES}

1. A. J. Wein and E. S. Rovner, Definition and epidemiology of overactive bladder, Urology 60 (2002) 7-12; DOI: 10.1016/S0090-4295(02)01784-3.

2. S. Tyagi, C. A. Thomas, Y. Hayashi and M. B. Chancellor, The overactive bladder: epidemiology and morbidity, Urol. Clin. North Am. 33 (2006) 433-438; DOI: 10.1016/j.ucl.2006.06.002.

3. H. Hashim and P. Abrams, Drug treatment of overactive bladder: Efficacy, cost and quality-of-life considerations, Drugs 64 (2004) 1643-1656; DOI: 10.2165/00003495-200464150-00003.

4. F. Haab, L. Stewart and P. Dwyer, Darifenacin, an $\mathrm{M}_{3}$ selective receptor antagonist, is an effective and well-tolerated once-daily treatment for overactive bladder, Eur. Urol. 45 (2004) 420-429; DOI: 10.1016/j.eururo.2004.01.008.

5. P. K. Kiptoo, M. O. Hamad, P. A. Crooks and A. L. Stinchcomb, Enhancement of transdermal delivery of 6-beta-naltrexol via a codrug linked to hydroxybupropion, J. Control. Release 113 (2006) 137-145; DOI: 10.1016/j.jconrel.2006.04.003.

6. K. Maman, S. Aballea, J. Nazir, K. Desroziers, M. E. Neine, E. Siddiqui, I. Odeyemi and Z. Hakimi, Comparative efficacy and safety of medical treatments for the management of overactive bladder: A systematic literature review and mixed treatment comparison, Eur. Urol. 65 (2014) 755-765; DOI: 10.1016/j.eururo.2013.11.010.

7. T. Omotosho and C. C. G. Chen, Update on tolterodine extended-release for treatment of overactive bladder, Open Access J. Urol. (2010) 185-191; DOI: 10.2147/OAJU.S7232.

8. P. Rama Rao, M. N. Reddy, S. Ramakrishna and P. V Diwan, Comparative in vivo evaluation of propranolol hydrochloride after oral and transdermal administration in rabbits, Eur. J. Pharm. Biopharm. 56 (2003) 81-85; DOI: 10.1016/S0939-6411(03)00038-9.

9. D. Clemett and B. Jarvis, Tolterodine: a review of its use in the treatment of overactive bladder, Drugs Aging 18 (2001) 277-304.

10. D. Gautam, T. S. Heard, Y. Cui, G. Miller, L. Bloodworth and J. Wess, Cholinergic stimulation of salivary secretion studied with M1 and M3 muscarinic receptor single- and double-knockout mice, Mol. Pharmacol. 66 (2004) 260-267; DOI: 10.1124/mol.66.2.260.

11. A. Arora, E. Kisak, P. Karande, J. Newsam and S. Mitragotri, Multicomponent chemical enhancer formulations for transdermal drug delivery: more is not always better, J. Control. Release 144 (2010) 175-180; DOI: 10.1016/j.jconrel.2010.02.015.

12. V. W. Nitti, S. Sanders, D. R. Staskin, R. R. Dmochowski, P. K. Sand, S. MacDiarmid and H. I. Maibach, Transdermal delivery of drugs for urologic applications: Basic principles and applications, Urology 67 (2006) 657-664; DOI: 10.1016/j.urology.2005.11.039. 
13. M. Akhlaq, M. S. Arshad, A. M. Mudassir, A. Hussain, I. Kucuk, R. Haj-Ahmad, M. Rasekh and Z. Ahmad, Formulation and evaluation of anti-rheumatic dexibuprofen transdermal patches: a quality-by-design approach, J. Drug Target. 24 (2016) 603-612; DOI: 10.3109/1061186X.2015.1116538.

14. L. Zhao, Y. Li, L. Fang, Z. He, X. Liu, L. Wang, Y. Xu and C. Ren, Transdermal delivery of tolterodine by O-acylmenthol: In vitro/in vivo correlation, Int. J. Pharm. 374 (2009) 73-81; DOI: 10.1016/j. ijpharm.2009.03.005.

15. F. Sun, C. Sui, Y. Zhou, X. Liu, Y. Shi, Y. Wu and Y. Li, Preparation, characterization and pharmacological evaluation of tolterodine hydrogels for the treatment of overactive bladder, Int. J. Pharm. 454 (2013) 532-538; DOI: 10.1016/j.ijpharm.2013.07.041.

16. A. H. Elshafeey, A. O. Kamel and M. M. Fathallah, Utility of nanosized microemulsion for transdermal delivery of tolterodine tartrate: ex-vivo permeation and in-vivo pharmacokinetic studies, Pharm. Res. 26 (2009) 2446-2453; DOI: 10.1007/s11095-009-9956-5.

17. R. Rajabalaya, S. R. David, J. Chellian, G. Xin Yun and S. Chakravarthi, Transdermal delivery of oxybutynin chloride proniosomal gels for the treatment of overactive bladder, Drug Deliv. 23 (2016) 1578-1587; DOI: 10.3109/10717544.2015.1116027.

18. R. Rajabalaya, G. Leen, J. Chellian, S. Chakravarthi and S. David, Tolterodine tartrate proniosomal gel transdermal delivery for overactive bladder, Pharmaceutics 8 (2016) 27; DOI: 10.3390/ pharmaceutics8030027.

19. R. Rajabalaya, S. R. N. David, J. Khanam and A. Nanda, Studies on the effect of plasticizer on in vitro release and ex vivo permeation from Eudragit E 100 based chlorpheniramine maleate matrix type transdermal delivery system, J. Excipients Food Chem. 1 (2010) 3-12.

20. S. R. N. David, M. S. Hui, C. F. Pin, F. Y. Ci and R. Rajabalaya, Formulation and in vitro evaluation of ethosomes as vesicular carrier for enhanced topical delivery of isotretinoin, Int. J. Drug Deliv. 5 (2013) 28-34.

21. T. Oki, A. Toma-Okura and S. Yamada, Advantages for transdermal over oral oxybutynin to treat overactive bladder: Muscarinic receptor binding, plasma drug concentration, and salivary secretion, J. Pharmacol. Exp. Ther. 316 (2006) 1137-1145; DOI: 10.1124/jpet.105.094508.

22. R. Rajabalaya, S. R. N. David, J. Khanam and A. Nanda, Effect of platicizers on in vitro release and ex vivo permeation of chlorpheniramine maleate from ethyl cellulose polyvinyl pyrrolidone based matrix patches, Farmacia 61 (2013) 975-990.

23. S. David, R. Rajabalaya and E. Zhia, Development and in vitro evaluation of self-adhesive matrixtype transdermal delivery system of ondansetron hydrochloride, Trop. J. Pharm. Res. 14 (2015) 211-218; DOI: 10.4314/tjpr.v14i2.4.

24. R. Rajabalaya, J. Khanam and A. Nanda, Design of a matrix patch formulation for long-acting permeation of diclofenac potassium, Asian J. Pharm. Sci. 3 (2008) 30-39.

25. R. Rajabalaya, T. W. Xian and S. R. N. David, Preparation and evaluation of transdermal drug delivery of ondansetron hydrochloride: effect of vegetable oils as permeation enhancer, Lat. Am. J. Pharm. 31 (2012) 1005-1012.

26. R. Rajabalaya, H. Q. Choo and S. R. N. David, Development and in vitro evaluation of matrix type transdermal delivery of ondansetron hydrochloride, Int. J. Drug Deliv. 4 (2012) 125-133.

27. J. Siepmann, F. Lecomte and R. Bodmeier, Diffusion-controlled drug delivery systems: calculation of the required composition to achieve desired release profiles, J. Control. Release 60 (1999) 379-389.

28. K. Ghosal, A. Chandra, R. Rajabalaya, S. Chakraborty and A. Nanda, Mathematical modeling of drug release profiles for modified hydrophobic HPMC based gels, Pharmazie 67 (2012) 147-155; DOI: 10.1691/ph.2012.1014.

29. T. Oki, S. Maruyama, Y. Takagi, H. I. Yamamura and S. Yamada, Characterization of muscarinic receptor binding and inhibition of salivation after oral administration of tolterodine in mice, Eur. J. Pharmacol. 529 (2006) 157-163; DOI: 10.1016/j.ejphar.2005.11.004. 